\title{
Global Aspects of Fixing the Gauge in the Polyakov String and Einstein Gravity
}

\section{T. P. Killingback ${ }^{\star}$}

University of Edinburgh, Department of Physics, James Clerk Maxwell Building, King's Buildings, Edinburgh EH9 3JZ, Scotland, UK

\begin{abstract}
It is shown that there exists a possible obstruction to a continuous global gauge choice in the Polyakov string theory and in four dimensional Einstein gravity. In many circumstances this obstruction results in no global gauge existing in these two theories.
\end{abstract}

\section{Introduction}

The Feyman path integral approach to quantising gauge theories appears to be the best method available at present. It has been applied with considerable success to the quantisation of Yang-Mills theories and QCD. In the Euclidean path integral approach to Yang-Mills theories one considers functional integrals of the form

$$
Z=\int_{\mathscr{C}} \mathscr{D} A \exp -S[A],
$$

where $\mathscr{D} A$ is a measure on the space $\mathscr{C}$ of all gauge potentials $A . S[A]$ is the YangMills action of $A$ and the functional integral is taken over all gauge potentials which satisfy some suitable boundary condition. However, it is well known that there is a problem in evaluating this path integral which results from the gauge invariance of the action $S[A]$.

Let $\mathscr{G}$ denote the group of gauge transformations. The difficulty arises because the orbits of $\mathscr{G}$ are expected to have infinite measure. The functional integral should really be carried out over the gauge orbit space $\mathscr{C} / \mathscr{G}$. However, $\mathscr{C} / \mathscr{G}$ is an intractable space. The idea of fixing the gauge is intended to circumvent this difficulty. We choose, in a continuous way, one gauge potential on each $\mathscr{G}$-orbit, i.e., we choose a continuous map $s: \mathscr{C} / \mathscr{G} \rightarrow \mathscr{C}$ such that $p \circ s=\mathrm{id}$, where $p: \mathscr{C} \rightarrow \mathscr{C} / \mathscr{G}$ is the canonical projection. The functional integral is then evaluated over $s(\mathscr{C} / \mathscr{G})$, weighted by the Jacobian of $p: s(\mathscr{C} / \mathscr{G}) \rightarrow \mathscr{C} / \mathscr{G}$. This yields the Fadeev-Popov

* Work supported by the Science and Engineering Research Council

Present address: Princeton University, Department of Physics, Joseph Henry Laboratories, Jadwin Hall, P.O. Box 708, Princeton, NJ 08544, USA 
determinant. As was shown in [1] the topologically non-trivial nature of the group $\mathscr{G}$ of gauge transformations results in the non-existence of a continuous global gauge for theories on the 4-sphere. In this paper we consider the possibility of choosing a global gauge fixing condition for theories which possess an invariance under the group of diffeomorphisms of a manifold $M$. The two theories of this type which we concentrate on are the Polyakov string and four dimensional Einstein gravity. It is shown that in both these theories there exists a topological obstruction to globally fixing the gauge. This obstruction comes from the topologically non-trivial nature of the group of diffeomorphisms of $M$. It is completely analogous to the obstruction to fixing the gauge in Yang-Mills theories discussed in [1]. It should be noted that similar ideas to those used here have also been discussed by Isham [2] in connection with the canonical quantisation of gravity.

The remainder of this paper is organized as follows. In Sect. 2 the formulation of the Polyakov string theory and four dimensional Euclidean gravity is recalled. Section 3 introduces the local gauge slices and the geometric structure of the orbit space of Riemannian metrics modulo diffeomorphisms. The obstruction to globally fixing the gauge in these two theories is then proved in Sect. 4.

\section{Formulation of the Polyakov String and Euclidean Gravity}

The dynamics of the Polyakov string is described in terms of the world sheet swept out by the string as it evolves in $D$ dimensional (Euclidean) space-time. The world sheet will be assumed to be a closed compact orientable 2 dimensional Riemannian manifold $M^{2}$. This 2 dimensional surface can be described by an immersion $x: M^{2} \rightarrow \mathbb{R}^{D}$, for $D \geqq 3$. Local charts on $M^{2}$ are $\left(U_{\alpha}, \xi_{\alpha}\right)$, where $U_{\alpha}$ is an open subset of $M^{2}$ and $\xi_{\alpha}$ a homeomorphism of $U_{\alpha}$ onto an open subset of $\mathbb{R}^{2}$. The immersion $x: M^{2} \rightarrow \mathbb{R}^{D}$ induces a Riemannian metric $h$ on $M^{2}$. Now let $g$ by a new Riemannian metric on $M^{2}$ independent of $h$. The local 2-form defined in the chart $\left(U_{\alpha}, \xi_{\alpha}\right)$ by

$$
\Omega_{(\alpha)}=\frac{1}{2} \operatorname{Tr}\left(g_{(\alpha)}^{-1} h_{(\alpha)}\right)\left(\operatorname{det} g_{(\alpha)}\right)^{1 / 2} d \xi_{(\alpha)}^{1} \wedge d \xi_{(\alpha)}^{2}=\frac{1}{2} \operatorname{Tr}\left(g_{(\alpha)}^{-1} h_{(\alpha)}\right) \mu(g),
$$

where $\mu(g)$ is the volume element associated to $g$, satisfies $\Omega_{(\alpha)}=\Omega_{(\beta)}$ in $U_{\alpha} \cap U_{\beta}$, and hence defines a global 2-form on $M^{2}$. The action of the string theory is defined to be (see [3])

$$
W[x, g]=\int_{M^{2}} \Omega=\frac{1}{2} \int_{M^{2}} \operatorname{Tr}\left(g^{-1} h\right) \mu(g) .
$$

This is the action introduced by Polyakov [4]. The partition function is then given by the path integral

$$
Z=\int \mathscr{D} g \mathscr{D} x \exp -W[x, g] .
$$

The Polyakov action is invariant under diffeomorphisms of $M^{2}$. Let $\mathscr{D}\left(M^{2}\right)$ denote the group of (orientation preserving) diffeomorphisms of $M^{2}$, and let $\mathscr{M}\left(M^{2}\right)$ denote the space of Riemannian metrics on $M^{2} . \mathscr{D}\left(M^{2}\right)$ acts naturally on $\mathscr{M}\left(M^{2}\right)$ by pull-back i.e., for $\pi \in \mathscr{D}\left(M^{2}\right)$ and $g \in \mathscr{M}\left(M^{2}\right)$ we have $g^{\pi}=\pi^{*} g \in \mathscr{M}\left(M^{2}\right)$. The diffeomorphism group of $M^{2}$ also acts on the immersion $x: M^{2} \rightarrow \mathbb{R}^{D}$ to give $x^{\pi}=x \circ \pi: M^{2} \rightarrow \mathbb{R}^{D}$. The metric induced on $M^{2}$ by the immersion $x^{\pi}$ is $h^{\pi}=\pi^{*} h$. 
The action $W[x, g]$ has the invariance $[4]$

$$
W\left[x^{\pi}, g^{\pi}\right]=W[x, g] .
$$

Therefore, two metrics on the same $\mathscr{D}\left(M^{2}\right)$-orbit correspond to the same geometry, but represented in two different sets of local charts. The space of inequivalent 2-geometries is given by the orbit space $\mathscr{M}\left(M^{2}\right) / \mathscr{D}\left(M^{2}\right)$.

A remarkable feature of the Polyakov string theory is that the partition function (2.3) can be evaluated explicitly (see [4]). To carry out the path integral over $\mathscr{D} g$ it is necessary to fix the gauge by choosing a representative metric of each orbit of $\mathscr{M}\left(M^{2}\right) / \mathscr{D}\left(M^{2}\right)$. Polyakov's choice was the conformal gauge, i.e., to find a representative of each orbit of the form (in a given local chart)

$$
g_{a b}(\xi)=e^{\phi(\xi)} \delta_{a b},
$$

which is always possible for 2 -dimensional surfaces. This choice will not uniquely specify the gauge if $M^{2}$ admits a non-trivial group $C\left(M^{2}\right)$ of conformal transformations,

$$
\begin{aligned}
C\left(M^{2}\right)= & \left\{\pi \in \mathscr{D}\left(M^{2}\right) \mid \text { there is a smooth } \varrho: M^{2} \rightarrow \mathbb{R}\right. \text { such that } \\
& \left.\pi^{*} g=e^{\varrho} g, \text { for all } g \in \mathscr{M}\left(M^{2}\right)\right\},
\end{aligned}
$$

(also see [3]). For $M^{2}=S^{2}, C\left(S^{2}\right)=\mathrm{SL}(2, \mathbb{C})$; for $M^{2}=T^{2}, C\left(T^{2}\right)=\mathrm{O}(2) \times \mathrm{O}(2)$; for surfaces of higher genus $C\left(M^{2}\right)$ is discrete (see [5]).

Using the conformal gauge the functional integral over $\mathscr{D} g$ can be evaluated to obtain the partition function as a functional integral over $\phi$ of the Liouville action

$$
L[\phi]=\int_{\mathcal{M}^{2}}\left[\frac{1}{2}\left(\partial_{a} \phi\right)^{2}+\mu^{2} e^{\phi}\right] d^{2} \xi .
$$

For this path integral over $\mathscr{D} \phi$ to be well defined it would seem to be necessary for the gauge choice (2.5) to be continuous. It is not clear that the conformal gauge satisfies this requirement. In fact, it will be shown later that for many surfaces $M^{2}$ there is no continuous global gauge fixing condition. This is a purely global result and there always exists a well defined local gauge.

Four dimensional Einstein gravity shares with the Polyakov string theory the property of being invariant under the diffeomorphism group of a compact Riemannian manifold. Let the (Euclidean) space-time be represented by a compact 4-dimensional Riemannian manifold $M^{4}$. The Euclidean action is (see [6])

$$
S[g]=-\frac{1}{16 \pi G} \int_{M^{4}} R(\operatorname{det} g)^{1 / 2} d^{4} x+\text { boundary term, }
$$

where $R$ is the scalar curvature of the metric $g$ on $M^{4}$. The manifolds with which we will be concerned will be closed and without boundary. Hence, we will neglect the boundary term in (2.7). If $\mathscr{M}\left(M^{4}\right)$ represents the space of Riemannian metrics on $M^{4}$ and $\mathscr{D}\left(M^{4}\right)$ the group of (orientation preserving) diffeomorphisms of $M^{4}$, then for $\pi \in \mathscr{D}\left(M^{4}\right)$ and $g \in \mathscr{M}\left(M^{4}\right)$,

$$
S\left[\pi^{*} g\right]=S[g] .
$$

Thus, the action is constant on orbits of $\mathscr{D}\left(M^{4}\right)$. 
The partition function is then defined to be

$$
Z=\int \mathscr{D} g \exp -S[g] \text {. }
$$

There are, of course, many problems in attempting to evaluate this functional integral (see [6]). In addition to these one might anticipate that the invariance of the action under the diffeomorphism of $M^{4}$ would lead to a similar problem in evaluating the path integral as occurs in gauge theories, i.e., that the orbits of $\mathscr{D}\left(M^{4}\right)$ would have infinite measure. The path integral should be carried out over the orbit space $\mathscr{M}\left(M^{4}\right) / \mathscr{D}\left(M^{4}\right)$, which is, however, intractable. This problem could be overcome by fixing the gauge [i.e., choosing in a continuous fashion a metric on each $\mathscr{D}\left(M^{4}\right)$-orbit] and then proceeding to evaluate the Fadeev-Popov determinant. We shall show later that for many 4-manifolds $M^{4}$ such a continuous global gauge does not exist. Local gauges, however, always exist and may be used to define the Fadeev-Popov determinant in a neighbourhood of a given metric $g \in \mathscr{M}\left(M^{4}\right)$.

\section{The Local Gauge Slices}

In this section we will consider the action of the group of diffeomorphisms of a compact manifold on the space of Riemannian metrics defined on the manifold. This topic has been investigated in detail by Ebin [7], and it has also been studied in connection with the theory of Wheeler-de Witt superspace [8]. Further details of the ideas mentioned here can be found in these references.

Let $M$ be a compact orientable $n$-dimensional manifold without boundary. $T M$ will be its tangent bundle, $T^{*} M$ its cotangent bundle and $S^{2} T^{*} M$ the bundle of symmetric covariant 2-tensors. The space of Riemannian metrics $\mathscr{M}(M)$ on $M$ is defined to be the space of all smooth sections of $S^{2} T^{*} M$ which induce a positive definite inner product on each tangent space $T_{x} M, x \in M$. Then $\mathscr{M}(M)$ is a positive open cone in $\Gamma\left(S^{2} T^{*} M\right)$, i.e., $\mathscr{M}(M)$ is open in $\Gamma\left(S^{2} T^{*} M\right)$ and if $\lambda, \mu>0$, $g, h \in \mathscr{M}(M)$, then $\lambda g+\mu h \in \mathscr{M}(M)$. Let $\mathscr{D}(M)$ be the group of orientation preserving diffeomorphisms of $M$. Then $\mathscr{D}(M)$ acts on $\Gamma\left(S^{2} T^{*} M\right)$ as follows: if $\pi \in \mathscr{D}(M), g \in \Gamma\left(S^{2} T^{*} M\right)$, and $X, Y \in T_{x} M, x \in M$, then

$$
\left(\pi^{*} g\right)_{x}(X, Y)=g_{\pi(x)}(T \pi X, T \pi Y)
$$

This action can be written as a map $A: \mathscr{D}(M) \times \Gamma\left(S^{2} T^{*} M\right) \rightarrow \Gamma\left(S^{2} T^{*} M\right)$. It is clear that $\mathscr{M}(M)$ is invariant under the action, so we can write $A: \mathscr{D} \times \mathscr{M} \rightarrow \mathscr{M}$. Note that when the manifold $M$ is not in question we will simplify $\mathscr{M}(M)$ and $\mathscr{D}(M)$ to $\mathscr{M}$ and $\mathscr{D}$, respectively.

The space of metrics has a (weak) Riemannian structure defined on it as follows. Each $h \in \mathscr{M}$ is a Riemannian structure on $T M$. It therefore induces a Riemannian structure on $T^{*} M$ and $S^{2} T^{*} M$. Let $(,)^{h}$ be this structure on $S^{2} T^{*} M$. Also $h \in \mathscr{M}$ induces a volume element $\mu$ on $M$. For $\omega, \theta \in \Gamma\left(S^{2} T^{*} M\right)$ we define $\langle\omega, \theta\rangle_{h}$ by

$$
\langle\omega, \theta\rangle_{h}=\int_{M}(\omega, \theta)^{h} \mu \text {. }
$$

$\langle,\rangle_{h}$ is a positive definite bilinear form on $\Gamma\left(S^{2} T^{*} M\right)$. Since $\mathscr{M}$ is open in $\Gamma\left(S^{2} T^{*} M\right), \mathscr{M}$ is a manifold whose tangent space at each point is canonically 
identified with $\Gamma\left(S^{2} T^{*} M\right)$. Thus for each $h \in \mathscr{M},\langle,\rangle_{h}$ on $\Gamma\left(S^{2} T^{*} M\right)$ defines a Riemannian structure on $\mathscr{M}$.

The most important property of $\langle,\rangle_{h}$ is that it is invariant under the natural action of $\mathscr{D}$ on $\mathscr{M}$, i.e., $\mathscr{D}$ acts by isometry. To see this first note that [7], for $\pi \in \mathscr{D}$, $g \in \mathscr{M}, \zeta, \xi \in T_{g} \mathscr{M} \simeq \Gamma\left(S^{2} T^{*} M\right), x \in M$,

$$
\left(\pi^{*} \zeta, \pi^{*} \xi\right)_{x}^{\pi^{*} g}=(\zeta, \xi)_{\pi(x)}^{g} .
$$

The diffeomorphism $\pi \in \mathscr{D}$ also acts on the set of volume elements of $M$ by pullback. If $\mu$ is the volume element of $g$ then $\pi^{*} \mu$ is the volume element of $\pi^{*} g$. Hence

or

$$
\int_{M}\left(\pi^{*} \zeta, \pi^{*} \xi\right)^{\pi^{*} g} \pi^{*} \mu=\int_{M}(\zeta, \xi)^{g} \mu
$$

$$
\left\langle\pi^{*} \zeta, \pi^{*} \xi\right\rangle_{\pi^{*} g}=\langle\zeta, \xi\rangle_{g}
$$

Therefore, $\pi \in \mathscr{D}$ is an isometry.

It is now possible to define the local gauge slice through a metric $g \in \mathscr{M}$ as the orthogonal complement of the tangent space to the $\mathscr{D}$-orbit through $g$, relative to the inner product $\langle,\rangle_{g}$. The orbit of $\mathscr{D}$ through $g \in \mathscr{M}$ is defined by the map

$$
\lambda_{g}: \mathscr{D} \rightarrow \mathscr{M}
$$

given by $\lambda_{g}(\pi)=\pi^{*} g$. The derivative of this map at the identity in $\mathscr{D}$ is

$$
T_{\mathrm{id}} \lambda_{g}: T_{\mathrm{id}} \mathscr{D} \rightarrow T_{g} \mathscr{M}
$$

Recalling the identifications $T_{\mathrm{id}} \mathscr{D} \simeq \Gamma(T M)$ and $T_{g} \mathscr{M} \simeq \Gamma\left(S^{2} T^{*} M\right)$, we have that

$$
T_{\mathrm{id}} \lambda_{g}: \Gamma(T M) \rightarrow \Gamma\left(S^{2} T^{*} M\right) .
$$

To compute $T_{\mathrm{id}} \lambda_{g}$, let $X \in \Gamma(T M)$ be a smooth vector field on $M . X$ generates a 1-parameter group of diffeomorphisms $\left\{\pi_{t}\right\}$. The smooth curve $C: \mathbb{R} \rightarrow \mathscr{D}$ in $\mathscr{D}$ given by $C(t)=\pi_{t}$, with $\pi_{0}=\mathrm{id}_{M}$, has tangent vector at $t=0$, given by $X=\left.(d / d t) C(t)\right|_{t=0} . T_{\text {id }} \lambda_{g}$ maps $X$ to the tangent vector to the curve $\left(\lambda_{g} \circ C\right)(t)$ in $\mathscr{M}$. Thus

$$
\begin{aligned}
\left(T_{\mathrm{id}} \lambda_{g}\right)(X) & =\left.\frac{d}{d t}\left(\lambda_{g} \circ C\right)(t)\right|_{t=0} \\
& =\left.\frac{d}{d t} \lambda_{g}\left(\pi_{t}\right)\right|_{t=0} \\
& =\left.\frac{d}{d t} \pi_{t}^{*} g\right|_{t=0} \\
& =\mathscr{L}_{X} g,
\end{aligned}
$$

where $\mathscr{L}_{X} g$ is the Lie derivative of $g$ with respect to the vector field $X$. Therefore the map $T_{\mathrm{id}} \lambda_{g}: \Gamma(T M) \rightarrow \Gamma\left(S^{2} T^{*} M\right)$ is given by the first order differential operator $\delta_{g}: \Gamma(T M) \rightarrow \Gamma\left(S^{2} T^{*} M\right)$, where

$$
\delta_{g} X=\mathscr{L}_{X} g
$$

for $X \in \Gamma(T M)$ and $g \in \mathscr{M}$. Note that $\operatorname{ker} \delta_{g}=$ space of Killing vectors on the Riemannian manifold $(M, g)$. 
It follows from standard properties of differential operators [9] that the image im $\delta_{g}$ is closed and has closed complement in $\Gamma\left(S^{2} T^{*} M\right)$. Thus the tangent space to the orbit through $g \in \mathscr{M}$ is given by $\operatorname{im} \delta_{g}$ and the local slice to this orbit at $g$ is the orthogonal complement of $\operatorname{im} \delta_{g}$ relative to the inner product $\langle,\rangle_{g}$. Hence, the local slice at $g \in \mathscr{M}$ is given by those $g+s \in \mathscr{M}, s \in \Gamma\left(S^{2} T^{*} M\right)$, for which

$$
0=\left\langle s, \delta_{g} X\right\rangle_{g}=\left\langle\delta_{g}^{*} s, X\right\rangle_{g}
$$

for all $X \in \Gamma\left(T^{*} M\right) . \delta_{g}^{*}$ is the $\langle,\rangle_{g}$-adjoint of $\delta_{g}$. The local slice through $g \in \mathscr{M}$ is written as

$$
\mathscr{S}_{g}=\left\{g+s \mid s \in \Gamma\left(S^{2} T^{*} M\right) \text { and } \delta_{g}^{*} s=0\right\} .
$$

In a sufficiently small neighbourhood of a given metric $g \in \mathscr{M}$ the slice $\mathscr{S}_{g}$ intersects each orbit once and only once [7]. Therefore this slice defines a good local gauge around $g$. We also have as a consequence of (3.5) the direct sum decomposition

$$
\Gamma\left(S^{2} T^{*} M\right) \simeq \operatorname{im} \delta_{g} \oplus \operatorname{ker} \delta_{g}^{*} .
$$

The geometrical structure of the orbit space $\mathscr{M} / \mathscr{D}$ will now be considered. The space $\mathscr{M} / \mathscr{D}$ is not a manifold because the action of $\mathscr{D}$ on $\mathscr{M}$ is not free. If we define the isometry group of $g \in \mathscr{M}$ to be

$$
I_{g}=\left\{\pi \in \mathscr{D} \mid \pi^{*} g=g\right\},
$$

then $\mathscr{D}$ has $g$ as a fixed point if $I_{g} \neq\{0\}$. There are, however, two ways to obtain a free action. If we restrict our attention to the space of metrics $\tilde{\mathscr{M}} \subset \mathscr{M}$ which have trivial isometry group, i.e.,

$$
\tilde{\mathscr{M}}=\left\{g \in \mathscr{M} \mid I_{g}=\{0\}\right\},
$$

then the action of $\mathscr{D}$ on $\tilde{\mathscr{M}}$ is free. The space $\tilde{\mathscr{M}}$ is open and dense in $\tilde{\mathscr{M}}$ [7]. The globally effective local slices $\mathscr{S}_{g}$ and the free $\mathscr{D}$-action on $\tilde{\mathscr{M}}$ results in a principal D-bundle

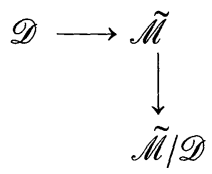

over $\tilde{\mathscr{M}} / \mathscr{D}$ which is now a smooth manifold.

Alternatively, we can restrict to the group $\mathscr{D}_{*}$ of diffeomorphisms which leave a point $x_{0} \in M$ fixed and also leave the frame at $x_{0}$ fixed, i.e.

$$
\mathscr{D}_{*}=\left\{\pi \in \mathscr{D} \mid \pi\left(x_{0}\right)=x_{0} \text { and } T_{x_{0}} \pi=\mathrm{id}_{T_{x_{0}} M}\right\} \text {. }
$$

Now if $\pi \in \mathscr{D}_{*}$ is an isometry for some $g \in \mathscr{M}$, i.e., if $\pi^{*} g=g$, then $\pi=\mathrm{id}_{M}$ (see [10]). Therefore, $\mathscr{D}_{*}$ acts freely on $\mathscr{M}$. Again the orbit space $\mathscr{M} / \mathscr{D}_{*}$ is a manifold and we have a principal $\mathscr{D}_{*}$-bundle over $\mathscr{M} / \mathscr{D}_{*}$

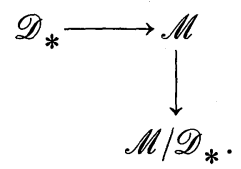

To conclude this section we make some statements of a geometrical and topological nature. 
Proposition 3.1. The manifolds $\tilde{\mathscr{M}} / \mathscr{D}$ and $\mathscr{M} / \mathscr{D} *$ have a natural Riemannian structure.

Proof. It follows from the invariance of $\langle,\rangle_{g}$ under the action of $\mathscr{D}$ on $\mathscr{M}$ [Eq. (3.2)] that $\langle,\rangle_{g}$ projects to give a well defined metric on $\tilde{\mathscr{M}} / \mathscr{D}$ and $\mathscr{M} / \mathscr{D}_{*}$.

Proposition 3.2. The space

$$
\mathscr{S}_{g}=\left\{g+s \mid s \in\left(S^{2} T^{*} M\right) \text { and } \delta_{g}^{*} s=0\right\}
$$

is the horizontal space at $g \in \tilde{\mathscr{M}}$ or $g \in \mathscr{M}$ of a connection on the bundle (3.8) or (3.9), respectively.

Proof. According to (3.7) the space $\mathscr{S}_{g}$ is complementary to the tangent space to the fibre at $g \in \mathscr{M}$. Since the Riemannian structure of $\mathscr{M}$ is preserved by the action of $\mathscr{D}$, we have that $\operatorname{ker} \delta_{\pi^{*} g}^{*}=\pi^{*}\left(\operatorname{ker} \delta_{g}^{*}\right)$, for all $\pi \in \mathscr{D}$. Therefore, $\mathscr{S}_{\pi^{*} g}=\pi^{*} \mathscr{S}_{g}$, and the slice $\mathscr{S}_{g}$ is the horizontal space of a connection.

Proposition 3.3. There are homotopy equivalences

$$
B \mathscr{D} \sim \tilde{\mathscr{M}} / \mathscr{D}
$$

and

$$
B \mathscr{D}_{*} \sim \mathscr{M} / \mathscr{D}_{*} \cdot
$$

Proof. These results follow from the contractibility of the total spaces of the bundles (3.8) and (3.9). The space of metrics $\mathscr{M}$ is convex and hence contractible. The space $\tilde{\mathscr{M}}$ is also contractible. This can be proved in analogy with Singer's proof (see [1]) that the space of irreducible connections on a principal SU(n)-bundle over a compact manifold is contractible.

Proposition 3.4. The groups $\mathscr{D}$ and $\mathscr{D}_{*}$ are related by the fibration

$$
0 \rightarrow \mathscr{D}_{*} \rightarrow \mathscr{D} \stackrel{\pi}{\longrightarrow} F_{+}(T M) \rightarrow 0,
$$

where $F_{+}(T M)$ is the principal $G L_{+}(n, \mathbb{R})$-bundle of frames on $M$ with a given orientation.

Proof. The projection $\pi$ is given by evaluation at the base-point. It is clear that the fibre of $\pi$ is $\mathscr{D}_{*}$ and that (3.10) has the homotopy lifting property.

\section{The Obstruction to Globally Fixing the Gauge}

We will now use the principal fibre bundles introduced in Sect. 3 to discuss the possibility of globally fixing the gauge in the Polyakov string theory and in four dimensional Euclidean gravity. Suppose that we are considering a theory defined on a compact orientable $n$-manifold $M^{n}$, with an action which is a $\mathscr{D}\left(M^{n}\right)$-invariant functional on $\mathscr{M}\left(M^{n}\right)$. Then a global gauge is a continuous map $s: \mathscr{M} / \mathscr{D} \rightarrow \mathscr{M}$ such that $p \circ s=\mathrm{id}_{\mathscr{M} / \mathscr{D}}$, where $p: \mathscr{M} \rightarrow \mathscr{M} / \mathscr{D}$ is the canonical projection. If such a gauge were to exist, then the restriction

$$
\left.s\right|_{\tilde{\mathscr{M}} \mid \mathscr{D}}: \tilde{\mathscr{M}} / \mathscr{D} \rightarrow \tilde{\mathscr{M}}
$$


would give a global section of the principal $\mathscr{D}$-bundle (3.8). Such a global section exists if and only if (3.8) is trivial, i.e.,

$$
\tilde{\mathscr{M}} \simeq \mathscr{D} \times \tilde{\mathscr{M}} / \mathscr{D} .
$$

Applying $\pi_{q}(\cdot)$ to this expression and recalling that $\tilde{\mathscr{M}}$ is contractible (see proof of Proposition 3.3), i.e. $\pi_{q}(\tilde{M})=0$, for all $q \geqq 0$, gives

$$
\pi_{q}(\mathscr{D}) \oplus \pi_{q}(\tilde{M} / \mathscr{D}) \simeq 0
$$

for all $q \geqq 0$. Thus, the obstruction to the existence of a global gauge is the nonvanishing of any of the homotopy groups of $\mathscr{D}$.

It should be noted that the bundle (3.8) has been used here purely as an auxillary device and has little direct physical significance. This is for the following reason. To obtain (3.8) it was necessary to restrict attention to those metrics with trivial isometry groups. However, it is known that many classical solutions of both the Polyakov string theory and four dimensional Euclidean gravity have nontrivial isometry groups. Therefore, to restrict attention to only those metrics in $\tilde{\mathscr{M}}$ eliminates many classical solutions which may be important in understanding the full theory. For example, such solutions may be required in order to undertake a semiclassical analysis of the theory.

In contradistinction to the unphysical nature of (3.8) the bundle (3.9) does have physical significance. The restriction to those diffeomorphisms of $M^{n}$ which leave both a point $x_{0} \in M^{n}$ fixed and the frame at $x_{0}$ fixed appears quite acceptable. For example, consider $M^{n}$ to be the one point compactification of a non-compact manifold $\hat{M}^{n}$, with $x_{0} \in M^{n}$ corresponding to the point at infinity in $\hat{M}^{n}$. Then the diffeomorphisms in $\mathscr{D}_{*}\left(M^{n}\right)$ correspond to the diffeomorphisms of $\hat{M}^{n}$ which are the identity at infinity and also have their derivative equal to the identity at infinity. This type of restriction on the diffeomorphisms of $\hat{M}^{n}$ is physically acceptable.

If we now consider fixing the gauge in a theory with a $\mathscr{D}_{*}\left(M^{n}\right)$-invariant action defined on $\mathscr{M}\left(M^{n}\right)$, then the bundle (3.9) may be used directly. In this case a global gauge choice is a global section of (3.9), which exists if and only if the bundle $\mathscr{M} \rightarrow \mathscr{M} / \mathscr{D}_{*}$ is trivial. By the same reasoning as used earlier, the obstruction to such a global section is the non-vanishing of any of the homotopy groups of $\mathscr{D}_{*}\left(M^{n}\right)$.

Taking the theory under consideration to be the Polyakov string, with $M^{2}$ a compact Riemann surface of genus $p$, invariant under either $\mathscr{D}\left(M^{2}\right)$ or $\mathscr{D}_{*}\left(M^{2}\right)$ yields the following results.

Theorem 4.1. There exists no global gauge $s: \mathscr{M} / \mathscr{D} \rightarrow \mathscr{M}$ for $M^{2}$ of genus $p=0$ or 1 . For $p>1$ there is no topological obstruction to the existence of such a gauge.

Proof. This follows directly from the homotopy type of $\mathscr{D}\left(M^{2}\right)$ [11], namely

$$
\begin{array}{lll}
\mathscr{D}\left(S^{2}\right) \sim \mathrm{SO}(3) & \text { for } & p=0 \\
\mathscr{D}\left(T^{2}\right) \sim \mathrm{SO}(2) \times \mathrm{SO}(2) & \text { for } & p=1 \\
\mathscr{D}\left(M^{2}\right) \sim\{0\} & \text { for } & p>1 .
\end{array}
$$

Theorem 4.2. There is no global section of $\mathscr{M} \rightarrow \mathscr{M} / \mathscr{D}_{*}$ for $M^{2}$ of genus $p>0$. For $p=0$ there is no obstruction to such a section. 
Proof. The homotopy groups of $\mathscr{D}_{*}\left(M^{2}\right)$ and $\mathscr{D}\left(M^{2}\right)$ are related by the exact homotopy sequence of the fibration (3.10)

$$
\ldots \rightarrow \pi_{q}\left(\mathscr{D}_{*}\left(M^{2}\right)\right) \rightarrow \pi_{q}\left(\mathscr{D}\left(M^{2}\right)\right) \rightarrow \pi_{q}\left(F_{+}\left(T M^{2}\right)\right) \rightarrow \pi_{q-1}\left(\mathscr{D}_{*}\left(M^{2}\right)\right) \rightarrow \ldots .
$$

Recall that $F_{+}\left(T M^{2}\right) \simeq \mathbb{R}^{3} \times \mathrm{O}\left(T M^{2}\right)$, where $\mathrm{O}\left(T M^{2}\right)$ is the principal $\mathrm{SO}(2)$ bundle of orthonormal frames of $M$. Hence, $F_{+}\left(T M^{2}\right)$ has the same homotopy type as $\mathrm{O}\left(T M^{2}\right)$. For $p=0, \mathscr{D}\left(S^{2}\right) \sim \mathrm{SO}(3)$ and $F_{+}\left(T S^{2}\right) \sim \mathrm{SO}(3)$ are isomorphic, hence

$$
\pi_{q}\left(\mathscr{D}_{*}\left(M^{2}\right)\right) \simeq 0
$$

for all $q \geqq 0$. This gives the last sentence of Theorem 4.2. For $p>0$, assume that

$$
\pi_{q}\left(\mathscr{D}_{*}\left(M^{2}\right)\right) \simeq 0
$$

for all $q \geqq 0$. It follows that

$$
\pi_{q}\left(\mathscr{D}\left(M^{2}\right)\right) \simeq \pi_{q}\left(F_{+}\left(T M^{2}\right)\right)
$$

for all $q \geqq 1$. Hence, for $p=1$ and $q=1,(4.1)$ implies that

$$
\pi_{1}(\mathrm{SO}(2) \times \mathrm{SO}(2)) \simeq \pi_{1}(\mathrm{SO}(2) \times \mathrm{SO}(2) \times \mathrm{SO}(2))
$$

i.e., $\mathbb{Z} \oplus \mathbb{Z} \simeq \mathbb{Z} \oplus \mathbb{Z} \oplus \mathbb{Z}$, which is a contradiction. For $p>1$, (4.1) implies that

$$
\pi_{q}\left(F_{+}\left(T M^{2}\right)\right) \simeq 0
$$

for all $q \geqq 1$. But from the defining fibration of $F_{+}\left(T M^{2}\right)$

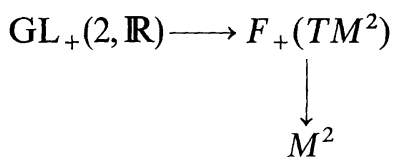

and (4.2) it follows that $\pi_{1}\left(M^{2}\right) \simeq 0$, which is a contradiction. Thus, $\mathscr{D}_{*}\left(M^{2}\right)$ is noncontractable for $M^{2}$ of genus $p>0$.

For four-dimensional Euclidean gravity, invariant under either $\mathscr{D}\left(M^{4}\right)$ or $\mathscr{D}_{*}\left(M^{4}\right)$, the obstruction to globally fixing the gauge is the non-contractability of $\mathscr{D}\left(M^{4}\right)$ or $\mathscr{D}_{*}\left(M^{4}\right)$, respectively. It is probable that for any compact 4-manifold $M^{4}$ the groups $\mathscr{D}\left(M^{4}\right)$ and $\mathscr{D}_{*}\left(M^{4}\right)$ will be homotopically non-trivial. For certain classes of compact 4-manifolds it is possible to show that $\pi_{0}\left(\mathscr{D}\left(M^{4}\right)\right) \neq\{0\}$ and $\pi_{0}\left(\mathscr{D}_{*}\left(M^{4}\right)\right) \neq\{0\}$. Note that it follows from the exact homotopy sequence of the fibration (3.10) that if $\pi_{0}\left(\mathscr{D}\left(M^{4}\right)\right) \neq\{0\}$ then $\pi_{0}\left(\mathscr{D}_{*}\left(M^{4}\right)\right) \neq\{0\}$.

The first class of compact 4-manifolds $M^{4}$ for which $\pi_{0}\left(\mathscr{D}\left(M^{4}\right)\right) \neq\{0\}$ are product manifolds.

Theorem 4.3. Let $M^{4}=N_{1} \times N_{2}$ (where $\operatorname{dim} N_{1}=\operatorname{dim} N_{2}=2$ ) be an oriented product manifold. Then

$$
\pi_{0}\left(\mathscr{D}\left(M^{4}\right)\right) \neq\{0\} .
$$

Proof. Let $\pi_{1}$ and $\pi_{2}$ be orientation reversing diffeomorphisms of $N_{1}$ and $N_{2}$ respectively. Let $\left[N_{1}\right] \in H^{2}\left(N_{1}\right)$ and $\left[N_{1}\right] \in H^{2}\left(N_{2}\right)$ be the 2 dimensional coho- 
mology classes of $N_{1}$ and $N_{2}$, respectively. Then $\pi_{1}^{*}\left[N_{1}\right]=-\left[N_{1}\right]$ and $\pi_{2}^{*}\left[N_{2}\right]$ $=-\left[N_{2}\right]$. Thus

$$
\begin{aligned}
\left(\pi_{1} \times \pi_{2}\right)^{*}\left[N_{1} \times N_{2}\right] & =\pi_{1}^{*}\left[N_{1}\right] \times \pi_{2}^{*}\left[N_{2}\right] \\
& =-\left[N_{1}\right] \times-\left[N_{2}\right] \\
& =\left[N_{1}\right] \times\left[N_{2}\right]=\left[N_{1} \times N_{2}\right]
\end{aligned}
$$

and $\pi_{1} \times \pi_{2}$ is an orientation preserving diffeomorphism of $N_{1} \times N_{2}$. However,

$$
\left(\pi_{1} \times \pi_{2}\right) *\left[N_{1}\right] \times 1=-\left[N_{1}\right] \times 1,
$$

and therefore $\pi_{1} \times \pi_{2}$ is not homotopic to the identity.

The second class of compact 4-manifolds which have a disconnected diffeomorphism group are smooth submanifolds of the complex projective space $\mathbb{P}^{3}$.

Theorem 4.4. Let $M^{4} \subset \mathbb{P}^{3}$ be a smooth compact 4-dimensional submanifold of $\mathbb{P}^{3}$. Then

$$
\pi_{0}\left(\mathscr{D}\left(M^{4}\right)\right) \neq\{0\} .
$$

Proof. Let $V \subset \mathbb{P}^{3}$ be a smooth 4-dimensional submanifold of $\mathbb{P}^{3}$ defined by the zero set of a polynomial $f$ with real coefficients

$$
V=\left\{x \in \mathbb{P}^{3} \mid f(x)=0\right\} .
$$

Let $c: \mathbb{P}^{3} \rightarrow \mathbb{P}^{3}$ given by $x \rightarrow \bar{x}$ be complex conjugation. Then $c$ sends $V$ to itself. If $a \in H^{2}\left(\mathbb{P}^{3}\right)$ is the positive generator of $H^{2}\left(\mathbb{P}^{3}\right)$, then $c^{*}: H^{2}\left(\mathbb{P}^{3}\right) \rightarrow H^{2}\left(\mathbb{P}^{3}\right)$ is given by $a \rightarrow-a$. Therefore, if $i: V \hookrightarrow \mathbb{P}^{3}$ is the inclusion of $V$ in $\mathbb{P}^{3},\left(\left.c\right|_{V}\right)^{*}$ sends $i^{*} a$ to $-i^{*} a$. Since $2 i^{*} a \neq 0$, it follows that $\left.c\right|_{V}$ is not homotopic to the identity. Since any $M^{4} \subset \mathbb{P}^{3}$ is diffeomorphic to a surface $V \subset \mathbb{P}^{3}$, it follows that

$$
\pi_{0}\left(\mathscr{D}\left(M^{4}\right)\right) \neq\{0\} \text {. }
$$

In the Euclidean approach to quantum gravity the compact 4-manifolds $S^{2} \times S^{2}, \mathbb{P}^{2}$ and the $K 3$ surface are important as compact gravitational instantons. It follows from Theorems 4.3 and 4.4 that the group of diffeomorphisms of these three manifolds is disconnected. Hence we have the following.

Theorem 4.5. Let $M^{4}$ be any one of the compact 4-manifolds $S^{2} \times S^{2}, \mathbb{P}^{2}$ or a K3 surface. Then

$$
\pi_{0}\left(\mathscr{D}\left(M^{4}\right)\right) \neq\{0\} .
$$

Proof. For $M^{4}=S^{2} \times S^{2}$ the result follows directly from Theorem 4.3 . For $M^{4}=\mathbb{P}^{2}$ the proof of Theorem 4.4 implies that $c: \mathbb{P} \rightarrow \mathbb{P}^{2}$ (complex conjugation) is not homotopic to the identity. The model of a $K 3$ surface is the quartic surface in $\mathbb{P}^{3}$ defined by

$$
\hat{M}=\left\{\left[x_{1}, x_{2}, x_{3}, x_{4}\right] \in \mathbb{P}^{3} \mid x_{1}^{4}+x_{2}^{4}+x_{3}^{4}+x_{4}^{4}=0\right\} .
$$

As $\hat{M}$ is defined by a polynomial with real coefficients, Theorem 4.4 implies that $\pi_{0}\left(\mathscr{D}\left(M^{4}\right)\right) \neq\{0\}$. As any $K 3$ surface $M^{4}$ is diffeomorphic to the surface $\hat{M}$, it follows that $\pi_{0}\left(\mathscr{D}\left(M^{4}\right)\right) \neq\{0\}$.

Furthermore, it follows from Theorem 4.5 that for $M^{4}=S^{2} \times S^{2}, \mathbb{P}^{2}$ or a $K 3$ surface

$$
\pi_{0}\left(\mathscr{D}_{*}\left(M^{4}\right)\right) \neq\{0\} .
$$


Therefore, it is impossible to globally fix the gauge in Euclidean gravity, with either a $\mathscr{D}\left(M^{4}\right)$ or $\mathscr{D}_{*}\left(M^{4}\right)$ invariance, defined on $S^{2} \times S^{2}, \mathbb{P}^{2}$ or a $K 3$ surface.

Although, as we have seen, in general it is not possible to define a global gauge in four dimensional Euclidean gravity, there always exist local gauges given by the gauge slices $\mathscr{S}_{g}$. These local gauges can be used to define the path integral in some sufficiently small neighbourhood $\mathscr{U}$ of $g \in \mathscr{M}$ in which $\mathscr{S}_{g}$ is a good gauge. We wish to evaluate

$$
Z=\int_{\mathscr{U}} \mathscr{D} g \exp -S[g] .
$$

If $\not: \mathscr{M} \rightarrow \mathscr{M} / \mathscr{D}_{*}$ is the canonical projection with the restriction $\left.\not\right|_{\mathscr{S}_{g}}: \mathscr{S}_{g} \rightarrow \mathscr{M} / \mathscr{D}_{*}$, then we can write

$$
\begin{aligned}
Z & =\int_{h_{1}\left(\mathscr{S}_{g}\right)} \mathscr{D} g \exp -S[g] \\
& =\int_{\mathscr{S}_{g}} \not||_{\mathscr{S}_{g}}^{*} \mathscr{D} g \exp -\mathscr{S}\left[\left.\not\right|_{\mathscr{S}_{g}} ^{*} g\right] \\
& =\int_{\mathscr{S}_{g}} \operatorname{det}\left(\left.\not\right|_{\mathscr{S}_{g}}\right) \mathscr{D} \hat{g} \exp -S[\hat{g}] .
\end{aligned}
$$

The Jacobian determinant $\operatorname{det}\left(\left.\not\right|_{\mathscr{g}_{g}}\right)$ of $\left.\not\right|_{\mathscr{S}_{g}}$ is the Fadeev-Popov determinant associated with the local gauge $\mathscr{S}_{g}$.

Acknowledgements. I would like to thank Elmer Rees for useful discussions concerning topology.

\section{References}

1. Singer, I.M.: Some remarks on the Gribov ambiguity. Commun. Math. Phys. 60, 7 (1978)

2. Isham, C.J.: Topological $\theta$-sectors in canonically quantized gravity. Phys. Lett. 106B, 188 (1981)

3. Onofri, E., Virasor, M.A.: On a formulation of Polyakov's string theory with regular classical solutions. Nucl. Phys. B 201, 159 (1982)

4. Polyakov, A.M.: Quantum geometry of bosonic strings. Phys. Lett. 103B, 207 (1981)

5. Goldberg, S.I.: Curvature and homology. New York: Academic Press 1962

6. Gibbons, G.W., Hawking, S.W., Perry, M.J.: Path integrals and the indefiniteness of the gravitational action. Nucl. Phys. B 138, 141 (1978)

7. Ebin, D.G.: In: Proc. Symposia in Pure Mathematics. Am. Math. Soc., Vol. 15, Providence, RI: AMS 1970

8. Fischer, A.E.: In: Relativity, Carmel, M., Fickler, S., Witten, L. (eds.). New York: Plenum 1967

9. Palais, R.S.: Seminar on the Atiyah-Singer index theorem. Princeton, NJ: Princeton University Press 1965

10. Helgason, S.: Differential geometry and symmetric spaces. New York: Academic Press 1962

11. Earle, C.J., Eells, J.: Bull. Am. Math. Soc. 73, 557 (1967)

Communicated by S. W. Hawking

Received October 8, 1984 
